\title{
Integrated Framework for Design and Testing of Software for Automotive Mechatronic Systems
}

\author{
Nick Van Kelecom ${ }^{1(\bowtie)}$, Timothy Verstraete $^{2}$, Sam Silverans ${ }^{1}$, \\ and Mathieu Dutré ${ }^{1}$ \\ ${ }^{1}$ Siemens Industry Software NV, Interleuvenlaan 68, 3001 Louvain, Belgium \\ \{nick.van_kelecom, sam. silverans, \\ mathieu.dutre\}@siemens.com \\ ${ }^{2}$ University of Antwerp, Groenenborgerlaan 171, 2020 Antwerp, Belgium \\ Timothy.Verstraete@uantwerpen. be
}

\begin{abstract}
Today's electrification and automation put a lot of pressure on automotive OEM's. Vehicle functions are increasingly executed by complex mechatronic systems, feeding the need for high-efficiency multi-disciplinary teams. Numerous car recalls confirm that establishing these teams is an unobvious process. A framework facilitating design, testing, engineering asset management and planning is essential. This research presents such an envisioned framework with a central development management tool, integrated with different engineering tools to obtain vital information.

First, high level requirements are defined, whereof a software architecture can be deduced. Based on this architecture, requirements can be remapped, refined and implemented. In parallel, test cases are written, verifying a requirement's implementation. Automatic test harness generation will reduce repetitive, timeintensive modelling. The testing status can later be centrally monitored. This status is the initiator for new engineering tasks, managed in a Kanban board providing a clear overview. Complete traceability through this process is ensured.
\end{abstract}

